J. Austral. Math. Soc. 21 (Series A) (1976), 371-375.

\title{
HOMOGENEOUS GRAPHS AND STABILITY
}

\author{
A. GARDINER \\ (Received 16 April 1975; revised 4 July 1975) \\ Communicated by W. D. Wallis
}

\begin{abstract}
Let $\Gamma$ be a graph with finite vertex set $V . \Gamma$ is homogeneous if whenever $U_{1}, U_{2} \subseteq V$ are such that the vertex subgraphs $\left\langle U_{1}\right\rangle,\left\langle U_{2}\right\rangle$ are isomorphic, then every isomorphism from $\left\langle U_{1}\right\rangle$ to $\left\langle U_{2}\right\rangle$ extends to an automorphism of $\Gamma$; homogeneous graphs were studied by Sheehan (1974) and were classified by the author. $\Gamma$ is locally homogeneous if whenever $U \subseteq V$, then every automorphism of $\langle U\rangle$ extends to an automorphism of $\Gamma$. We prove that every locally homogeneous graph is homogeneous.
\end{abstract}

We study finite, undirected, loopless graphs $\Gamma=(V, E)$, with vertex set $V=V \Gamma$, edge set $E \subseteq V \times V$, and automorphism group Aut $\Gamma=G$. If $U \subseteq V$, then the vertex subgraph $\langle U\rangle$ has vertex set $U$ and edge set $(U \times U) \cap E$. We have a natural metric $\partial$ on $V$ and denote by $d$ the diameter of $\Gamma$. Set

$$
\Gamma_{i}:=\{(u, v) \in V \times V: \partial(u, v)=i\}, \quad 0 \leqq i,
$$

and for $u \in V$

$$
\Gamma_{i}(u):=\left\{v \in V:(u, v) \in \Gamma_{i}\right\} .
$$

We write $\Gamma(u):=\Gamma_{1}(u)$.

$K_{r}$ denotes the complete graph on $r$ vertices, $K_{k, k}$ denotes the complete bipartite graph of valency $k, K_{t: r}$ denotes the complete $t$-partite graph with blocks of size $r, C_{n}$ denotes the circuit of length $n, 0_{3}$ denotes Petersen's graph. (These graphs are described in Wilson (1972), to which the reader is referred for the general graph theoretical background.)

If $\Gamma$ is a graph, then $t \cdot \Gamma$ denotes the disjoint union of $t$ copies of $\Gamma, \Gamma^{c}$ denotes the complement of $\Gamma$, and $L(\Gamma)$ denotes the line graph of $\Gamma$.

If $U \subseteq V$, then $G_{U}$ and $G_{t U]}$ denote respectively the pointwise and setwise stabilisers of $U$; if $U=\left\{u_{1}, u_{2}, \cdots, u_{i}\right\}$, then we simply write $G_{u_{1} u_{2} \cdots u_{i}}$ and $G_{\left[u_{1}, u_{2}, \cdots, u_{i}\right] .} S_{t}$ denotes the symmetric group on $t$ symbols. Basic facts about permutation groups can be found in Wielandt (1964). 
A graph is homogeneous if whenever $\left\langle U_{1}\right\rangle,\left\langle U_{2}\right\rangle$ are isomorphic subgraphs of $\Gamma$ each isomorphism from $\left\langle U_{1}\right\rangle$ to $\left\langle U_{2}\right\rangle$ extends to an automorphism of $\Gamma$. Finite homogeneous graphs were studied by Sheehan (1974) and were classified by the present author.

A graph is locally homogeneous if whenever $U \subseteq V$, each isomorphism from $\langle U\rangle$ to $\langle U\rangle$ extends to an automorphism of $\Gamma$. Clearly $\Gamma$ is locally homogeneous if and only if $\Gamma^{c}$ is locally homogeneous. Each homogeneous graph is locally homogeneous by definition. We prove the converse by classifying locally homogeneous graphs.

THEOREM. A finite locally homogeneous graph is homogeneous.

Let $\Gamma$ be a graph and let $v_{1}, v_{2}, \cdots, v_{t} \in V$; then we define $\Gamma_{v_{1}}:=\left\langle V-\left\{v_{1}\right\}\right\rangle$, and for each $i, 1 \leqq i<t, \Gamma_{v_{1} v_{2} \cdots v_{i+1}}:=\left(\Gamma_{v_{1} v_{2} \cdots v_{i}}\right)_{v_{i+1}}$.

A graph $\Gamma$ is stable if for some enumeration $\left(v_{1}, v_{2}, \cdots, v_{n}\right)$ of the vertex set $V, G_{v_{1} v_{2} \cdots v_{i}}=\operatorname{Aut}\left(\Gamma_{v_{1} v_{2} \cdots v_{i}}\right)$ for each $i, 1 \leqq i \leqq n . \Gamma$ is totally stable if for each enumeration $\left(v_{1}, v_{2}, \cdots, v_{n}\right)$ of the vertex set $V, G_{v_{1} v_{2} \cdots v_{i}}=$ Aut $\left(\Gamma_{v_{1} v_{2} \cdots v_{1}}\right)$ for each $i, 1 \leqq i \leqq n$.

COROllary [Yap (1974) Theorem 4]. The only totally stable graphs are the complete and the null graphs.

We assume throughout that $\Gamma$ is some locally homogeneous graph.

LEMMA 1. If $\Gamma$ is disconnected, then $\Gamma \cong t \cdot K$, for some $t, r \geqq 1$.

Proof. Let $V=U_{1} \cup U_{2} \cup \cdots \cup U_{t}, t \geqq 2$, be the decomposition of $V$ into connected components. Choose $u_{i} \in U_{i}, 1 \leqq i \leqq t$; then $\left\langle u_{1}, u_{2}, \cdots, u_{t}\right\rangle \cong$ $t \cdot K_{1}$, so $G_{\left[u_{1}, u_{2}, \cdots, u_{t}\right]}$ induces the full symmetric group $S_{t}$ on $\left\langle u_{1}, u_{2}, \cdots, u_{t}\right\rangle$. Thus all the connected components of $\Gamma$ are isomorphic. If $\left\langle U_{1}\right\rangle$ is not a complete graph choose $u_{0}, u_{1}$ such that $u_{0} \in \Gamma_{2}\left(u_{1}\right)$; then $G_{\left[u_{0}, u_{1}, \cdots, u_{t}\right]}$ induces the full symmetric group $S_{t+1}$ on $\left\langle u_{0}, u_{1}, \cdots, u_{t}\right\rangle \cong(t+1) \cdot K_{1}$. Hence $t=1$.

Lemma 2. If $\Gamma$ is connected, then $(G, V)$ is transitive and either $\Gamma \cong K_{k+1}$, or $d=2$.

Proof. If $u \in V, v \in \Gamma(u)$, then $G_{[u, v]}$ induces the symmetric group $S_{2}$ on $\langle u, v\rangle$. Thus for each $u \in V, v \in \Gamma(u), G$ contains an element $g_{v}$ for which $u^{\boldsymbol{g}_{v}}=v$; since $\Gamma$ is connected, $(G, V)$ must be transitive. In particular $|\Gamma(u)|=k$ and $\left|\Gamma_{2}(u)\right|=k_{2}$ are independent of $u \in V$. If $d=1$, then $\Gamma \cong K_{k+1}$. Assume $d \geqq 2$ and choose $u \in V, v \in \Gamma_{2}(u)$. If $d=4$, choose $w \in \Gamma_{4}(u)$; then $G_{[u, v, w]}$ must induce the full symmetric group $S_{3}$ on $\langle u, v, w\rangle$, which is evidently impossible. Suppose $d=3$. If we can choose $w \in \Gamma_{3}(u)-\Gamma(v)$, then we obtain a contradiction as for $d=4$. Thus $\Gamma_{3}(u) \subseteq \Gamma(v)$ for each $v \in \Gamma_{2}(u), \Gamma_{2}(u) \subseteq \Gamma(w)$ 
for each $w \in \Gamma_{3}(u)$; then $\Gamma_{2}(w) \supseteq \Gamma(u)$, so since $(G, V)$ is transitive we have $\Gamma_{2}(u)=\Gamma(w), \Gamma(u)=\Gamma_{2}(w)$, and $\Gamma_{3}(u)=\{w\}$. But then for $x \in \Gamma(u), \Gamma_{3}(x)=$ $\{y\} \subseteq \Gamma_{2}(u),\langle u, x, y\rangle=K_{2} \cup K_{1}$. and no element of $G_{\{u, x, y]}$ can fix $y$ and interchange $u \in \Gamma_{2}(y)$ and $x \in \Gamma_{3}(y)$. Thus $d=2$.

Lemma 3. If $\Gamma$ has diameter $d=2$, then for each $u \in V, G_{u}$ acts transitively on $\Gamma(u)$ and on $\Gamma_{2}(u)$ (in other words: $\Gamma$ is a rank three graph).

PRoof. Let $v \in \Gamma(u)$. For each $w \in \Gamma(v) \cap \Gamma(u), G_{[u, v \cdot w]}$ induces the full symmetric group $S_{3}$ on $\langle u, v, w\rangle$ so $G_{u}$ contains an element interchanging $v$ and $w$. Thus $G_{u}$ acts transitively on each connected component of $\langle\Gamma(u)\rangle$. Further if $v_{1}, v_{2}$ lie in distinct connected components of $\langle\Gamma(u)\rangle$, then $G_{\left\{u, v 1, v_{2}\right]}$ contains an element fixing $u$ and interchanging $v_{1}$ and $v_{2}$. Hence $G_{u}$ acts transitively on the connected components of $\langle\Gamma(u)\rangle$. The result for $\Gamma_{2}(u)$ follows by considering the complement of $\Gamma$.

Thus we may assume that $\Gamma$ is a connected (rank three) graph of diameter $d=2$.

Lemma 4. $\langle\Gamma(u)\rangle$ is locally homogeneous.

Proof. Let $U \subseteq \Gamma(u)$. Then each automorphism $\varphi$ of $\langle U\rangle$ corresponds to a unique automorphism $\hat{\varphi}$ of $\langle U \cup\{u\}\rangle$ fixing $u$, and $\hat{\varphi}$ extends to an automorphism of $\Gamma$ (fixing $u$ ) which leaves $\Gamma(u)$ invariant. Thus $\varphi$ extends to an automorphism of $\langle\Gamma(u)\rangle$.

Thus if $\Gamma$ is locally homogeneous we may choose $u \in V=V \Gamma$ and pass to the locally homogeneous graph $\Gamma^{1}:=\langle\Gamma(u)\rangle$, then choose $u_{1} \in V \Gamma^{1}$ and pass to the locally homogeneous graph $\Gamma^{2}:=\left\langle\Gamma^{1}\left(u_{1}\right)\right\rangle$, etc., until we finally obtain some graph $\Gamma^{i}$ isomorphic to $t \cdot K_{r}$ for some $t, r$. We must thus determine the minimal class of graphs which contains all the graphs $t \cdot K_{r}$ and which is closed with respect to 'extension'. Let $u \in V$.

LEMMA 5. If $\langle\Gamma(u)\rangle \cong k \cdot K_{1}, k \geqq 2$, then $\Gamma \cong C_{3}$ or $\Gamma \cong K_{k, k}$.

Proof. We assume $k \geqq 3, d=2$. For each $w \in \Gamma_{2}(u),|\Gamma(w) \cap \Gamma(u)|=c_{2}$ is constant. If $c_{2}=1$, then $\Gamma$ is a Moore graph admitting a rank three group, and so is either $0_{3}$ or the Hoffman-Singleton graph; however $0_{3}$ contains a vertex subgraph isomorphic to $3 \cdot K_{2}$ on which Aut $0_{3} \cong S_{5}$ does not induce the full wreath product $S_{2} \backslash S_{3}$, and the Hoffman-Singleton graph has vertex stabiliser $G_{u} \cong S_{7}$ whereas if $v \in \Gamma(u)$, then $G_{u v}$ induces $S_{k-1} \times S_{k-1}$ on $\Gamma(u) \cup \Gamma(v)$. If $k>c_{2} \geqq 2$, then $G_{((u) \cup r(u)]}$ induces the full symmetric group $S_{k}$ on $\Gamma(u)$ so each $c_{2}$-subset of $\Gamma(u)$ corresponds to a vertex of $\Gamma_{2}(u)$ and

$$
\left(\begin{array}{l}
k \\
c_{2}
\end{array}\right) \leqq k_{2}=\frac{k(k-1)}{c_{2}} \text {. }
$$


Moreover if $x \in \Gamma(w) \cap \Gamma_{2}(u)$, then $\Gamma(x) \cap \Gamma(u) \cap \Gamma(w)=\varnothing$, so $2 c_{2} \leqq k$. Hence $c_{2}=2$. $G_{[\{u, w\} \cup(\Gamma(u)-\Gamma(w)])}$ induces the full symmetric group $S_{k-2}$ on $\Gamma(u)-\Gamma(w)$ so each 2-subset of $\Gamma(u)-\Gamma(w)$ corresponds to a vertex of $\Gamma(w) \cap \Gamma_{2}(u)$. Thus $\left(\begin{array}{c}k-2 \\ 2\end{array}\right)=k-2$, so $k=5,|V|=16,\left\langle\Gamma_{2}(u)\right\rangle \cong 0_{3}$, and $0_{3}$ is not locally homogeneous. Thus $c_{2}=k, \Gamma \cong K_{k, k}$.

Lemma 6. If $\langle\Gamma(u)\rangle \cong K_{k}$, then $\Gamma \cong t \cdot K_{k+1}$, for some $t$.

Lemma 7. If $\langle\Gamma(u)\rangle \cong t \cdot K_{r}, r \geqq 2, t \geqq 2$, then $\Gamma \cong L\left(K_{3,3}\right)$.

Proof. Let $\Gamma(u)=U_{1} \cup U_{t} \cup \cdots \cup U_{\mathrm{t}}$ be the decomposition of $\langle\Gamma(u)\rangle$ into connected components. If $v \in \Gamma_{2}(u)$, then $\left|\Gamma(v) \cap U_{i}\right| \leqq 1$, so $c_{2} \leqq t$. $G_{[\{u\} u r(u)]}$ induces the full wreath product $S_{r} \backslash S_{t}$ on $\Gamma(u)$. Thus each subgraph $c_{2} \cdot K_{1}$ of $\langle\Gamma(u)\rangle$ corresponds to some vertex of $\Gamma_{2}(u)$. Hence

$$
\left(\begin{array}{c}
t \\
c_{2}
\end{array}\right) r^{c_{2}} \leqq k_{2}=\frac{\operatorname{tr}(t r-r)}{c_{2}},
$$

so $c_{2} \leqq 2$. On the other hand $\Gamma(u) \cap \Gamma_{2}(v)$ contains a subset $U$ with $\langle U\rangle=$ $t \cdot K_{r-1}$, so $G_{[\{u, v\} \cup U]}$ induces the full wreath product $S_{r-1} \mid S_{t}$ on $U$ and fixes both $u$ and $v$. Hence $t=c_{2}=2, \Gamma$ is a line graph, $\Gamma=L(\Delta)$, and $\Delta$ is bipartite of diameter two and valency $r+1$. Hence $\Gamma \cong L\left(K_{r+1, r+1}\right)$. If $s \geqq 4$, then $L\left(K_{s, s}\right)$ contains a vertex subgraph $2 \cdot C_{4}$ on which the full automorphism group $\left.D_{8}\right\} S_{2}$ is not induced. Hence $r=2=t, \Gamma \cong L\left(K_{3.3}\right)$.

Lemma 8. If $\langle\Gamma(u)\rangle \cong K_{t ; r}, r \geqq 2, t \geqq 2$, then $\Gamma \cong K_{t+1 ; r}$.

Proof. Choose $v \in \Gamma(u)$ and set $\Gamma(v) \cap \Gamma_{2}(u)=: W$. In $\langle\Gamma(v)\rangle \cong K_{t: r}$ we see that $\langle\{u\} \cup W\rangle \cong r \cdot K_{1}$ and that for each $v_{1} \in \Gamma(u) \cap \Gamma(v), W \subseteq \Gamma\left(v_{1}\right)$. Since $\langle\Gamma(u)\rangle$ is connected we obtain $W=\Gamma_{2}(u), \Gamma \cong K_{t+1 ; r}$.

LEMMA 9. $\langle\Gamma(u)\rangle \not C_{5}, L\left(K_{3,3}\right)$.

Proof. Suppose $\langle\Gamma(v)\rangle \cong C_{5}$ for each $v \in V$. Considering each $v \in \Gamma(u)$ in turn forces $\left\langle\Gamma_{2}(u)\right\rangle \cong C_{5}$, whence $\Gamma$ is the isosahedron, contrary to $d=2$. Suppose $\langle\Gamma(u)\rangle \cong L\left(K_{3,3}\right)$. Choose $w \in \Gamma_{2}(u)$. If $v \in \Gamma(u) \cap \Gamma(w)$, then $\mid \Gamma(u) \cap$ $\Gamma(v) \cap \Gamma(w) \mid=2$, and applying this to $v_{1} \in \Gamma(u) \cap \Gamma(w) \cap \Gamma(v)$ implies $c_{2} \geqq 4$. Since $9 \cdot 4 / c_{2}=k_{2}$ we have either (a) $c_{2}=4$, or (b) $c_{2}=6$. If $c_{2}=4$, then $|V|=19$, so $\Gamma$ is a graph of valency 9 on 19 vertices which is impossible. If $c_{2}=6$, then $\left\langle\Gamma_{2}(u)\right\rangle$ is a trivalent graph on six vertices, so $\left\langle\Gamma_{2}(u)\right\rangle \cong K_{3.3}$. But $\Gamma^{c}$ then contradicts Lemma 7.

Our induction is thus complete: a finite locally homogeneous graph is one of the following: (i) $t \cdot K_{r}, t \geqq 1, r \geqq 1$, (ii) $K_{t ; r}, t \geqq 1, r \geqq 1$, (iii) $C_{5}$, (iv) $L\left(K_{3.3}\right.$ ). But each of these graph is also homogeneous. Thus we have the 
THEOREM. The following conditions on a finite graph $\Gamma$ are equivalent:

(a) $\Gamma$ is homogeneous,

(b) $\Gamma$ is locally homogeneous,

(c) $\Gamma$ is one of the graphs $t \cdot K_{r}, t \geqq 1, r \geqq 1 ; K_{t, r}, t \geqq 1, r \geqq 1 ; C_{5} ; L\left(K_{3,3}\right)$.

Remark. Our results do not in fact need the full force of the finiteness assumption; the same results, with the same proofs, hold for locally finite graphs.

\section{References}

A. Gardiner (to appear), 'Homogeneous graphs', J. Combinatorial Theory.

J. Sheehan (1974), 'Smoothly embeddable subgraphs', J. London Math. Soc. (2), 9, 212-218.

R. J. Wilson (1972), Introduction to graph theory (Oliver and Boyd, Edinburgh).

H. Wielandt (1964), Finite permutation groups (Academic Press, New York).

H. P. Yap (1974), 'Some remarks on stable graphs', Bull. Austral. Math. Soc. 10, 351-357.

Pure Mathematics

University of Birmingham

Birmingham B15 2TT

England. 